\title{
Analysis of the Relationship of Blood Metabolites with White Blood Cells in Periparturient Dairy Cattle
}

\author{
Lea Logan ${ }^{\mathrm{a}}$, Aridany Suarez-Trujillo ${ }^{\mathrm{a}}$, Karen Plaut ${ }^{\mathrm{a}}$, Theresa Casey ${ }^{\mathrm{a}}{ }^{*}$
}

\begin{abstract}
Periparturient dairy cattle are susceptible to metabolic disease due to the immense physiological stress caused by calving and the onset of lactation. Susceptibility to disease is related to the cow's ability to maintain metabolic homeostasis and mount a proper immune response, despite high demand for glucose to support fetal growth and milk synthesis, and as a fuel for increased circulating levels of white blood cells (WBC). This study was conducted to determine if there was a correlation among white blood cell percentages, blood glucose, and the levels of the ketone betahydroxybutyrate (BHBA) on the days immediately around parturition, and to determine if the variables exhibited circadian rhythms. We hypothesized that glucose levels would vary inversely with WBC, BHBA levels would vary inversely with glucose levels, and BHBA levels would vary directly with WBC. Blood was sampled from the tail vein of multiparous Holstein cows $(n=12)$ at 0600 from 4 days prepartum to 4 days postpartum, and every 4 hr over a $24-$ $\mathrm{hr}$ period $(\mathrm{n}=6)$ starting at 0600 on postpartum day 3. Blood glucose and BHBA levels were measured using a CentriVet ${ }^{\mathrm{TM}}$, and WBC percentage was calculated by measuring packed cell volume. Glucose was higher $(p<0.05)$ before $(73.77 \mathrm{mg} / \mathrm{dL} \pm 4.65)$ versus after $(68.85 \mathrm{mg} / \mathrm{dL} \pm 6.15)$ parturition, whereas BHBA was higher $(p<0.05)$ after calving $(0.53 \mathrm{mg} / \mathrm{dL} \pm 0.39)$ versus before $(0.20$ $\mathrm{mg} / \mathrm{dL} \pm 0.16)$. WBC percentages did not vary significantly by day. None of the variables exhibited circadian rhythms according to cosinor analysis. Pearson's correlation analysis revealed an inverse relationship $(r=-0.23, p=0.04)$ between glucose and BHBA levels, and a direct relationship between WBC percentage and BHBA $(r=0.25, p=0.03)$. This finding supported our hypothesis, and suggests that immune responses during the periparturient period may affect metabolic homeostasis.
\end{abstract}

Keywords: dairy cattle, transition period, glucose, ketones, white blood cell

The transition period in dairy cattle is the period spanning three weeks prepartum to three weeks postpartum. This period encompasses the end of the cow's non-lactating period, which takes place approximately 45-60 days prior to expected calving; parturition; and the onset of lactation. During early lactation, approximately one-third of cows will contract a metabolic or infectious disease. The increased susceptibility to disease is related to her ability to adapt to changes in physiological demands, i.e. maintain homeostasis, and mount proper immune responses. Metabolic disease and overall poor health during the transition period can impair milk production over the whole length of lactation (LeBlanc, 2010). Throughout the transition period, there is a high demand on the body for energy and nutrients needed to support fetal growth and the onset of synthesis of milk. However, due to the size of the fetus and physiological changes associated with parturition cows, feed intake decreases. This causes the cow to enter a negative energy balance (NEB) (Wankhade et al., 2017).

The body responds to alleviate the NEB by mobilizing body fat stores as non-esterified fatty acids. NEFA are metabolized in the liver during gluconeogenesis and ketone bodies including beta-hydroxybutyrate (BHBA) are released as a byproduct. BHBA levels increase as a cow's NEB increases. If BHBA levels rise above $1.2 \mathrm{mmol} / \mathrm{L}$, the cow is in a ketotic state, which is associated with lethargy, a further decrease in feed intake, and decreased milk production (LeBlanc, 2010).

The immune system becomes more active during the transition period in part by proliferation of several different subclasses. White blood cells require glucose as a primary fuel for survival and immune function. White blood cells have glucose transporters (Glut1) that allow glucose uptake into the cell, and the expression of those transporters is regulated by extracellular signals such as hormones or cytokines (Maciver et al., 2008) that are released during the periparturient period.
Therefore, as their populations increase, demand for glucose increases. However, cows often experience anorexia during times of heightened immune activity. During active infection, milk production often decreases, so glucose can be spared for the immune system (Esposito et al., 2014), which can drive cows further into a NEB.

The body maintains a relatively constant level of internal conditions called homeostasis, which the onset of metabolic disease can disrupt. Metabolic homeostasis is regulated in part by the circadian system (Gnocchi and Bruscalupi, 2017). The circadian system functions by aligning the body's physiological and behavioral processes through the generation of circadian rhythms. A circadian rhythm is a biological process that oscillates over a $24-\mathrm{hr}$ period of time and is synchronized to the body's external environment and nutritional cues. The suprachiasmatic nucleus, which is the body's master clock, is located in the anterior hypothalamus. It gathers external cues, such as light and dark cycles, integrates it, and conveys information to peripheral clocks located in various tissues throughout the body, such as the mammary gland. The molecular clocks primarily function via highly coordinated transcription-translation feedback systems to generate circadian rhythms (Eckel-Mahan and Sassone-Corsi, 2013).

Blood glucose and ketone levels are regulated in part by the circadian timing system, and levels were shown to exhibit circadian rhythms in mid-lactation dairy cows (Niu and Harvatine, 2018). Our previous studies found multiparous dairy cattle exposed to chronic phase-shifts in the light-dark cycle, a chronic jet-lag model, had significantly decreased glucose levels relative to control cows. There was no significant difference between treatments in the weight of the calves at birth, so it is unlikely that the depressed glucose levels in the phase-shifted group came from increased glucose

a. Department of Animal Sciences, Purdue University, West Lafayette, IN, 47907

*Corresponding author: Theresa Casey, Research Associate Professor, Biochemistry 326, 175 S. University, West Lafayette,

IN, 47907. Email: theresa-casey@ purdue.edu Phone: 802-373-1319 
requirements of the fetus during gestation. Additionally, both groups of cows exhibited periparturient surges in circulating levels of glucose within hours of calving. Circulating glucose then returned to steady state levels by day 3 postpartum, although levels were significantly lower than the prepartum period, indicating homeostasis was intact (Suarez-Trujillo et al., 2020). We hypothesized that the lower blood glucose levels in the phase-shifted group from our previous study were due to effects of circadian system disruption on the immune system. Studies of humans and rodents found the immune system is regulated by circadian clocks, and circulating white blood cells exhibit circadian rhythms (Labrecque and Cermakian, 2015). However, little is known regarding the role of circadian rhythms during the transition period in dairy cows.

Here, we conducted a study to test the hypothesis that as white blood cell percentages increase, glucose levels will decrease during the transition period. Moreover, since ketone levels will likely increase as glucose levels decrease, we anticipated ketone levels will be higher with higher white blood cell percentages. The overall objective of the study was to determine if there was a correlation among white blood cell percentages, blood glucose, and BHBA levels in cows in days immediately around parturition. Additionally, cows were blood sampled every $4 \mathrm{hr}$ over $24 \mathrm{hr}$ to determine if white blood percentages, blood glucose, and ketone levels exhibited circadian rhythms on day 3 postpartum. Although the relationships of these specific blood metabolites are widely known, this study allowed us to directly examine the variables in the days immediately around parturition, which is not extensively studied in dairy cattle. Additionally, there are very few studies that aimed to capture circadian rhythms of blood metabolites and percent white blood cells at three days postpartum, when we predict animals would be returned to homeostasis.

\section{Materials and Methods}

\section{Animals}

Prior to experimentation, all animal-related experimental protocols were reviewed and approved by Purdue University's Institutional Animal Care and Use Committee. The study included 12 multiparous Holstein dairy cattle that entered the study at three days prior to their expected calving date. Cows were housed individually in box stalls where they received a regular schedule of 16 hours of light and $8 \mathrm{hr}$ of darkness. They were fed a pre-fresh diet leading up to parturition, and were switched to a lactation diet immediately upon calving. The composition of the diets is described in Table 1.
Table 1. Ingredients and nutrient composition of pre-fresh and lactating diets.

\begin{tabular}{lcc}
\hline Item & Pre-fresh Diet & Lactating Diet \\
\hline Ingredient (\% of DM) & & \\
Corn silage & 32.7 & 24.6 \\
Alfalfa silage & 5.4 & 19.1 \\
Rye hay & 16.2 & 7.8 \\
Ground corn & 14.4 & 4.8 \\
High moisture shell corn & & 17.4 \\
Soybean meal & 6.4 & 5.5 \\
Soyplus & 3.6 & 4.8 \\
Whole fuzzy cottonseed & & 6.0 \\
Cottonseed hulls & 6.6 & \\
Distillers grains with solubles & 0.7 & \\
LysAAMet & 0.6 & 0.8 \\
QLF Blend 63-38 & 2.3 & 3.7 \\
Calcium carbonate & 1.2 & 1.1 \\
Palmit 80 & & 1.3 \\
Biochlor & 7.0 & \\
Vitamin and mineral mix & 2.91 & $3.1^{2}$ \\
Nutrient composition (\% of DM) & & \\
DM & 57.0 & 49.9 \\
NDF & 33.2 & 28.6 \\
CP & 15.8 & 15.6 \\
NFC & 39.6 & 42.2 \\
FA & 3.9 & 5.4 \\
\hline
\end{tabular}

1 Pre-fresh vitamin and mineral mix contained $28.55 \%$ MegalacR, $13.25 \%$ Diamond V XP, $13.96 \%$ magnesium oxide, $9.11 \%$ monocalcium phosphate, $8.54 \%$ magnesium sulfate, $7.32 \%$ salt, $6.68 \%$ Vitamin E 20,000, $6.07 \%$ calcium sulfate, $5.94 \%$ mineral premix, $0.43 \%$ Rumensin $90 \mathrm{~g} / \mathrm{lb}$, and $0.15 \%$ Vitamin A 30,000.

${ }^{2}$ Lactating vitamin and mineral mix contained $27.07 \%$ sodium bicarbonate, $13.60 \%$ salt, $10.89 \%$ monocalcium phosphate, $10.72 \%$ DCAD Plus, $6.68 \%$ Omnigen AF, $6.40 \%$ Diamond V XP, $6.59 \%$ magnesium oxide, $5.99 \%$ calcium sulfate, $5.90 \%$ fat yellow grease, $3.38 \%$ mineral premix, $1.80 \%$ ground corn, $0.78 \%$ Vitamin E 20,000 and $0.21 \%$ Rumensin 90g/lb.

All of the cows had natural births. One cow was treated for ketosis ; no data was collected from this cow following initiation of treatment.

\section{Blood sampling}

To capture changes in glucose, BHBA and white blood cell (WBC) volume across the transition period, blood samples $(\sim 2 \mathrm{ml})$ were taken one time daily at 0600 from the coccygeal tail vein using a 20 -gauge needle. For temporal analysis of circadian rhythms, blood samples were taken from 6 animals every four hours for 24 hours beginning at 0600 on the third day following parturition.

\section{Glucose and ketone analysis}

Glucose and BHBA levels were measured with a CentriVet $^{\mathrm{TM}}$ (ACON Laboratories, California) using whole blood.

\section{Evaluation of white blood cell percentage}

White blood cell percentage was measured using hematocrit analysis. Briefly, blood was drawn into glass microhematocrit capillary tubes (Fisherbrand ${ }^{\mathrm{TM}}$ Microhematocrit Capillary Tubes, 2019), and centrifuged. Total length, 
including plasma, red blood cell, and white blood cell length; red blood cell and white blood cell lengths combined; and red blood cell length were measured using a digital caliper. To determine the length of the white blood cell layer, the measurement of the red blood cell layer was subtracted from measurement of the red and white blood cell layer. Then, the white blood cell layer length was divided by the total length to determine the percentage of white blood cells. When white blood cell volume was below the detectable level of the instrument, a value of $0.5 \mathrm{x}$ the lowest WBC length recorded across all of the cows was entered for that time point.

\section{Statistical analysis}

Descriptive statistics, including mean and standard deviation, for each factor were calculated using Excel. Data were presented as mean \pm standard deviation (S.D.). A general linear model (two-way ANOVA) analysis and t-test analysis were performed in Mini Tab 18. Cow and time point were the two factors in ANOVA analysis. A $p$-value less than 0.05 was considered statistically significant. If the factor was considered statistically significant, pair-wise Tukey's was performed. Pearson's linear regression analysis was used to determine if there was a relationship between dependent variables. Cosinor analysis of data collected every $4 \mathrm{hr}$ over $24 \mathrm{hr}$ was performed using RStudio (RStudio 1.1.453, Boston, MA) to determine if it fit a 24-hr rhythm. Posthoc analysis of sample size was run using the following tool (https://www.dssresearch.com/resources/calculators/statistical -power-calculator-average/).

\section{Results}

\section{Glucose levels relative to parturition}

To describe changes in glucose levels across the days immediately surrounding parturition, samples were taken at 0600 daily in cows ( $n=12$; Figure 1$)$. Day relative to parturition had a significant effect on glucose levels $(p<0.001)$. Cow was not a significant factor in glucose levels. Post-hoc Tukey analysis indicated day 0PP, which is the day of parturition, was significantly different both to days prior to parturition (BC) and days following parturition (PP). A t-test was performed and indicated that average glucose level across the days prior ( $73.77 \mathrm{mg} / \mathrm{dL} \pm 4.65$ ) to parturition was higher than the average across the days following $(68.85 \mathrm{mg} / \mathrm{dL} \pm 6.15)$ parturition $(p=0.030)$.

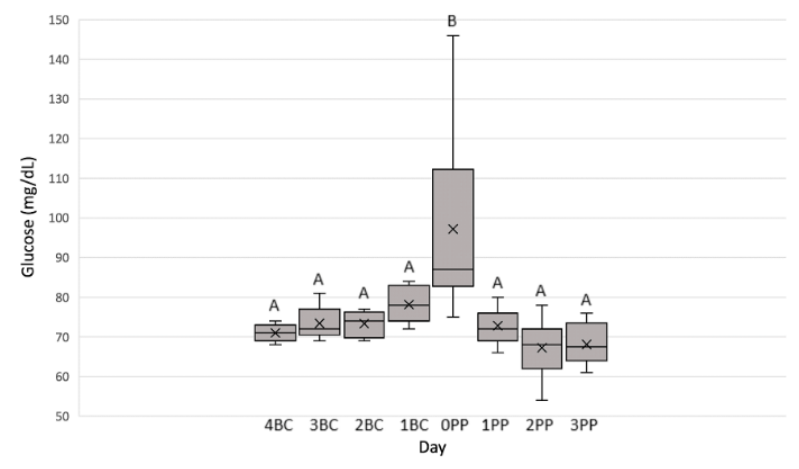

Figure 1. Glucose level on day relative to parturition. Whole blood glucose levels were measured at 0600 daily in cows $(n=12)$. Two-way ANOVA indicated day had an overall effect $(p<0.05)$ on blood glucose. According to Post-hoc Tukey's analysis, any day not sharing a common label (A, B, or C) is significantly different $(p<0.05)$. Data are presented as mean \pm S.D.

\section{Ketone levels relative to parturition}

Ketone levels were measured as BHBA in $\mathrm{mg} / \mathrm{dL}$ at 0600 daily in cows ( $n=12$; Figure 2), and two-way ANOVA found levels different among days relative to parturition $(p<0.001)$. Additionally, ketone levels differed significantly among the cows. Post-hoc Tukey analysis indicated days two, three, and four before parturitions to be significantly lower than days 1 , 2 , and 3 post-partum.

Student t-test analysis indicated the average BHBA level across the days prior $(0.20 \mathrm{mg} / \mathrm{dL} \pm 0.16)$ to calving was significantly lower than the average across the days after $(0.53$ $\mathrm{mg} / \mathrm{dl} \pm 0.39)$ calving $(p<0.001)$

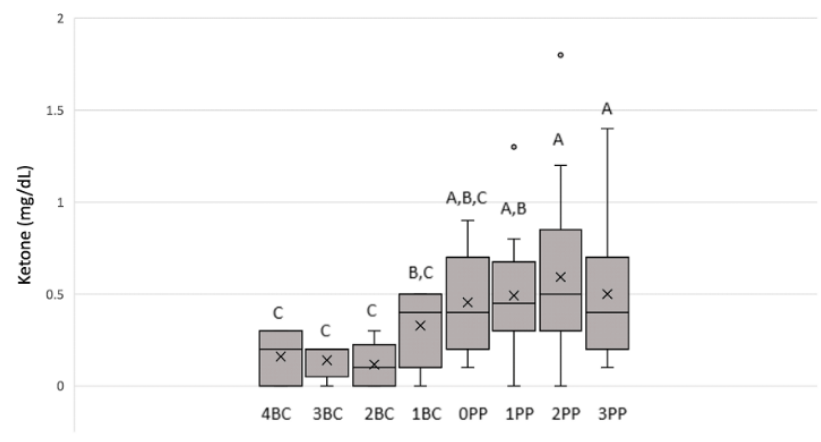

Day

Figure 2. Ketone level on day relative to parturition. Whole blood ketone levels were measured at 0600 daily in cows $(n=12)$. Two-way ANOVA indicated day had an overall effect $(p<0.05)$ on ketone levels. According to Post-hoc Tukey's analysis, any day not sharing a common label (A, B, or C) is significantly different $(p<0.05)$. Data are presented as mean \pm S.D.

\section{White blood cell percentages relative to parturition}

White blood cell percentages were measured at 0600 daily in cows $(\mathrm{n}=11$; Figure 3$)$ using hematocrit analysis. Differences among white blood cell percentages were not found statistically significant based on day relative to parturition or based on cow. Moreover, t-test analysis indicated mean percent before and after parturition were not statistically different $(p=0.41)$.

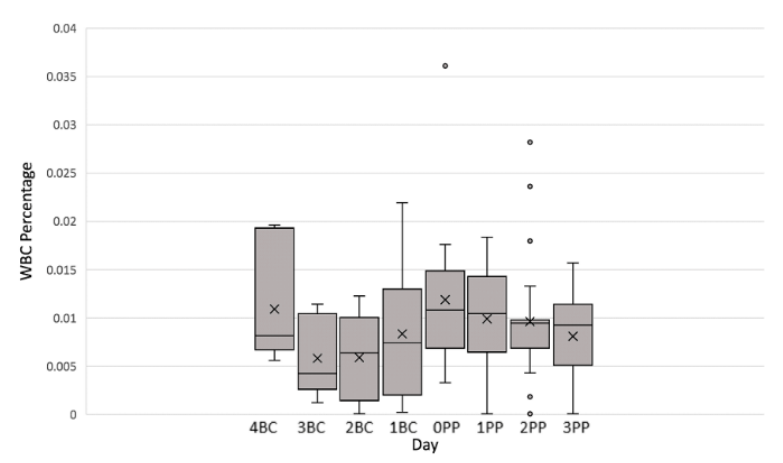


Figure 3. White blood cell percentage on day relative to parturition. White blood cell percentages were measured at 0600 daily in cows using hematocrit analysis $(n=11)$. Twoway ANOVA indicated day and cow had no overall effect $(p<0.05)$ on white blood cell percentage. Data are presented as mean \pm S.D.

\section{Glucose levels across circadian time points}

Whole blood glucose levels were measured every four hr starting at 0600 on day 3PP and ending at 0600 on day 4PP $(n=6$; Figure 4$)$ to capture changes over a $24-\mathrm{hr}$ period. Cosinor analysis indicated the data did not fit a 24-hr rhythm $(p>0.05)$.

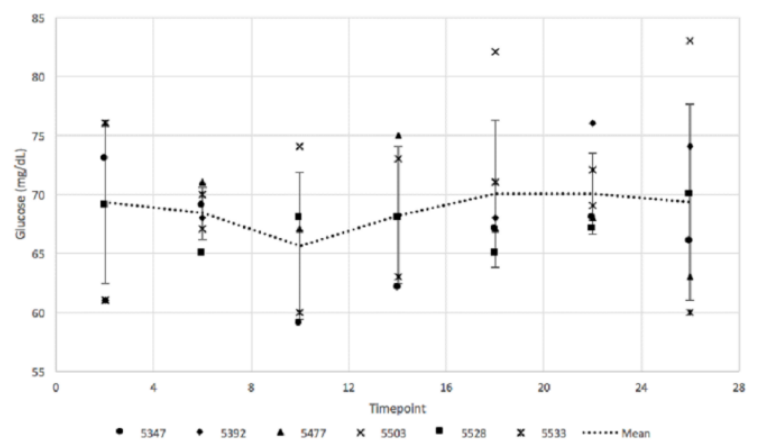

Figure 4. Glucose levels across circadian time points. Whole blood glucose levels were measured every four hours starting at 0600 on day 3PP and ending at 0600 on day 4PP $(n=6)$. The line indicates the mean \pm S.D across all cows.

\section{Ketone levels across circadian time points}

Ketone levels were measured every four hr starting at 0600 on day $3 \mathrm{PP}$ and ending at 0600 on day 4PP $(\mathrm{n}=6$; Figure 5). Cosinor analysis indicated the data did not fit a 24-hr rhythm $(p>0.05)$.

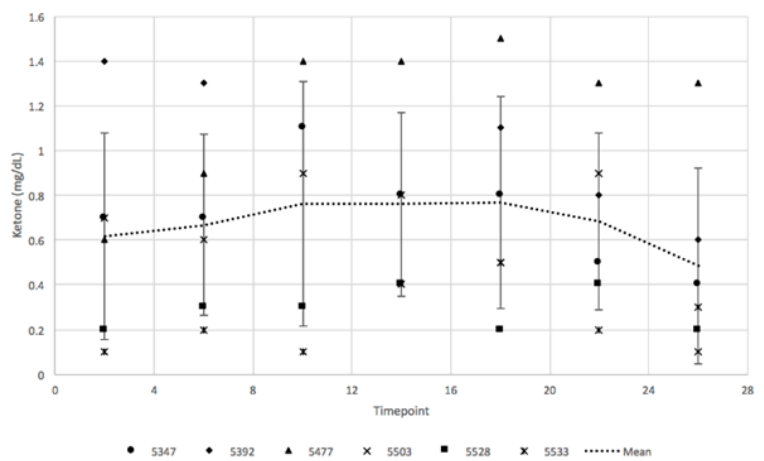

Figure 5. Ketone levels across circadian time points. Whole blood ketone levels were measured every four hours starting at 0600 on day 3PP and ending at 0600 on day 4PP $(n=6)$. The line indicates the mean \pm S.D across all cows.

\section{White blood cell percentages across circadian time points}

White blood cell percentages were measured using hematocrit analysis every four hr starting at 0600 on day 3PP and ending at 0600 on day 4PP ( $\mathrm{n}=6$; Figure 6). Cosinor analysis indicated the data did not fit a $24-\mathrm{hr}$ rhythm.

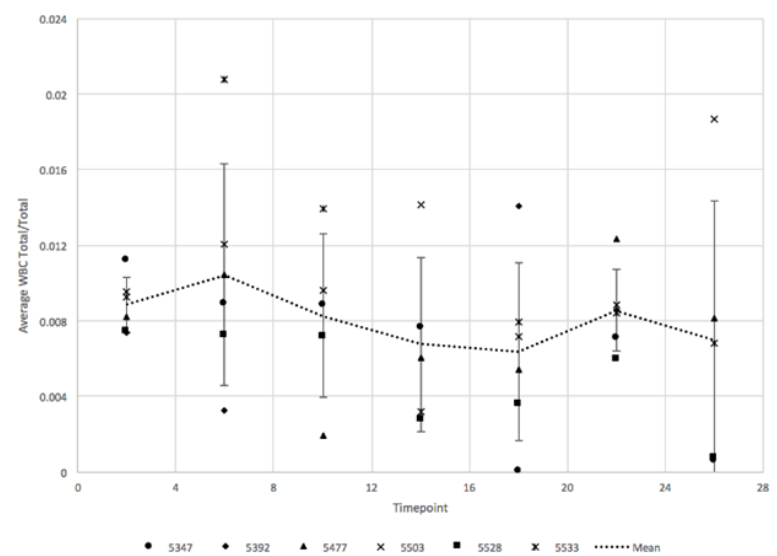

Figure 6. White blood cell percentages across circadian time points. White blood cell percentages were measured every four hours starting at 0600 on day 3PP and ending at 0600 on day $4 \mathrm{PP}(\mathrm{n}=6)$ The line indicates the mean \pm S.D across all cows.

\section{Correlation analysis of the relationship of blood metabolites with WBC percentage}

Pearson's correlation analysis was performed to determine if there was a relationship between white blood cell percentages, blood glucose, and BHBA levels (Table 2). Blood glucose levels taken at 0600 were negatively related $(p<0.05)$ to blood BHBA levels, and WBC percent was positively related to 0600 BHBA levels.

Table 2. Pearson's correlation analysis of the relationship of blood metabolites and WBC percentage.

\begin{tabular}{|lcc|}
\hline Comparison & Pearson correlation (r) & $p$-value \\
\hline Transition period sampling ( $\mathrm{n}=12$ ) & & \\
\hline WBC percentage versus glucose & -0.105 & 0.384 \\
Glucose versus BHBA & -0.229 & $0.041^{*}$ \\
WBC percentage versus BHBA & 0.256 & $0.031^{*}$ \\
\hline Circadian sampling (n=6) & & \\
\hline WBC percentage versus glucose & 0.255 & 0.117 \\
\hline Glucose versus BHBA & -0.122 & 0.459 \\
\hline WBC percentage versus BHBA & -0.158 & 0.332 \\
\hline
\end{tabular}

\section{Discussion}

Measuring glucose provides an indication of the cow's metabolism. In this study, glucose levels were significantly higher on the day of parturition than days before and after. This was predicted because of the known relationship between fetal cortisol release at parturition and increased gluconeogenesis (Gross et al., 2015). Cortisol among other hormones is released during calving as part of the fetal and maternal stress response (Vannucchi et al., 2015). Cortisol activates key enzymes involved in glucose production in the liver and inhibits glucose uptake in peripheral tissues, which leads to an increased overall concentration of blood glucose in the mother on the day of parturition (Marik and Bellomo, 2013). Additionally, glucose levels were higher pre-partum versus postpartum. This was expected and is due to lactation being more energetically demanding than the growth of the fetus. Compared to the gravid uterus on day 250 of pregnancy; the mammary gland on lactation day 4 takes up three-times more glucose to support milk synthesis (Bell, 1995). 
As glucose levels decreased, ketones levels increased from pre- to postpartum sampling periods, so glucose and ketone levels displayed a negative correlation across the transition period samples. Cows reduce feed intake around the time of parturition and enter a negative energy balance. In response to negative energy balance, fat is mobilized from adipose tissue and reflected in increased circulating levels of non-esterified fatty acids (NEFA). NEFAs are used as substrates for gluconeogenesis in the liver, with BHBA released as a by-product of this process.

White blood cells percent was measured as an indicator of the activity of the immune system; a higher white blood cell percentage would signify a more active immune system. Percent white blood cells did not differ by day nor between preand postpartum periods. The method used in collecting the white blood cell data was susceptible to human error, as several students were collecting data using a digital caliper that is sensitive to slight movement, which may have led to the high degree of variability in data. Moreover, a previous study of immune cell population changes in transition period dairy cows found that the different types of immune cells vary in number across the transition period (Todorović and Davidović, 2012). In particular, numbers of neutrophils were greatest on the day of parturition, and significantly lower 15 days before and after calving. Lymphocyte concentrations were lowest on the day of parturition, and increased slightly following calving. Monocyte concentrations remained relatively constant during pregnancy, and were lower during the last month of pregnancy and parturition. Basophil and eosinophil concentration remained constant during the transition period. Thus, populations may have changed in our study, but percent circulating of all white blood cell types may not be different. Lack of a difference in WBC percentages may have also been limited by sample size; post-hoc analysis using mean and standard deviation of white blood cell percentage on days $0 \mathrm{PP}$ and 3PP indicated a sample size of 14 would be needed to detect a potential difference between days. Though dependent on availability, there are several automated hematology analyzers that can produce highly accurate complete blood cell counts using bovine parameters that perhaps would have been the ideal method to provide a precise value for white blood cell counts (Pieper et al., 2016).

Percent white blood cells were related to BHBA levels. A study of transition period dairy cows found that cows with subclinical ketosis showed a heightened immune response compared with metabolically healthy individuals (Schulz et al., 2015). Therefore, the positive correlation among ketone levels and white blood cell percentages is consistent with these findings.

Circadian samples were collected on the third day following parturition, which immediately follows the large physiological change of calving and the onset of lactation. Therefore, it is highly likely that normal circadian rhythms of blood metabolites and immune cells were masked by the dramatic changes in metabolism that occurred in the cows (Rietveld et al., 1993).

This study served to evaluate efficacy of approach for studying the relationship between blood metabolites and circulating white blood cells. The design of future studies will need to consider all factors including sample size and the methods used to measure variables. Ideally, more cows would need to be included in the study, and less students should measure white blood cell percentages in order to reduce potential technical variation in sample analysis. Despite challenges presented within the study design, this study effectively evaluated critical health indicators in dairy cows across the days immediately surrounding parturition.

Our hypothesis was widely supported, with WBC varying directly with BHBA levels, and glucose levels varying inversely with BHBA levels. These relationships reflect the relationship between a cow entering a negative energy balance due to lack of glucose and the subsequent release of ketone bodies as a byproduct of NEFAs being metabolized in the liver. As a cow undergoes the immense physiological changes associated with the transition period, she is more likely to contract a metabolic disease, therefore heightening the immune system, and is more likely to decrease her feed intake, making her more susceptible for entering a NEB. Analysis of glucose, white blood cells, and BHBA provided further knowledge of how their relationships vary at the time of calving and the onset of lactation.

\section{Acknowledgments}

This work was supported by the Agriculture and Food Research Initiative (AFRI) competitive grant no. 2017-6701526569 project accession no. 1011965 from the USDA National Institute of Food and Agriculture. Data were collected with the assistance of the following students: Megan Brown, Sarah Luecke, Emilee Ludwick, Samantha Hartman-Stackhouse, Kirsten Senn, Kelsey Teeple, Grace Wernert, and Haley Westfall. We thank the students for their contributions, and we also thank the staff members at the Purdue Animal Sciences Research and Education Center Dairy Unit for their cooperation and assistance.

\section{References}

Bell, A. W. 1995. Regulation of organic nutrient metabolism during transition from late pregnancy to early lactation. J Anim Sci 73(9):2804-2819.

Eckel-Mahan, K. and P. Sassone-Corsi. 2013. Metabolism and the circadian clock converge. Physiol Rev 93(1):107-135.

Esposito, G., P. C. Irons, E. C. Webb, and A. Chapwanya. 2014. Interactions between negative energy balance, metabolic diseases, uterine health and immune response in transition dairy cows. Anim Reprod Sci 144(3-4):60-71.

Fisherbrand ${ }^{\text {TM }}$ Microhematocrit Capillary Tubes. 2019. Page www.fischersci.com. Fisher Scientific.

Gnocchi, D. and G. Bruscalupi. 2017. Circadian Rhythms and Hormonal Homeostasis: Pathophysiological Implications. Biology (Basel) 6(1).

Gross, J. J., O. Wellnitz, and R. M. Bruckmaier. 2015. Cortisol secretion in response to metabolic and inflammatory challenges in dairy cows. J Anim Sci 93(7):3395-3401.

Labrecque, N. and N. Cermakian. 2015. Circadian Clocks in the Immune System. J Biol Rhythms 30(4):277290.

LeBlanc, S. 2010. Monitoring metabolic health of dairy cattle 
Research Article (2019)

in the transition period. J Reprod Dev 56 Suppl:S29-35.

Maciver, N. J., S. R. Jacobs, H. L. Wieman, J. A. Wofford, J. L. Coloff, and J. C. Rathmell. 2008. Glucose metabolism in lymphocytes is a regulated process with significant effects on immune cell function and survival. J Leukoc Biol 84(4):949-957.

Marik, P. E. and R. Bellomo. 2013. Stress hyperglycemia: an essential survival response! Crit Care 17(2):305.

Niu, M. and K. J. Harvatine. 2018. The effects of feeding a partial mixed ration plus a top-dress before feeding on milk production and the daily rhythm of feed intake and plasma hormones and metabolites in dairy cows. J Dairy Sci 101(1):164-171.

Pieper, I. L., Y. Friedmann, A. Jones, and C. Thornton. 2016. Evaluation of Four Veterinary Hematology Analyzers for Bovine and Ovine Blood Counts for In Vitro Testing of Medical Devices. Artif Organs 40(11):1054-1061.

Rietveld, W. J., D. S. Minors, and J. M. Waterhouse. 1993. Circadian rhythms and masking: an overview. Chronobiol Int 10(4):306-312.

Schulz, K., J. Frahm, S. Kersten, U. Meyer, D. Reiche, H. Sauerwein, and S. Dänicke. 2015. Effects of elevated parameters of subclinical ketosis on the immune system of dairy cows: in vivo and in vitro results. Arch Anim Nutr 69(2):113-127.

Suarez-Trujillo, A., G. Wernert, H. Sun, T. S. Streckler, K. Huff, S. Cummings, J. Franco, J. R. Townsend, M. Grott, J. S. Johnson, K. Plaut, J. P. Boerman, and T. M. Casey. 2020. Exposure to chronic light-dark phase shifts during the prepartum nonlactating period attenuates circadian rhythms, decreases blood glucose, and increases milk yield in the subsequent lactation. 103(3):2784-2799.

Todorović, M. J. and V. Davidović. 2012. Changes in white blood pictures and some biochemical parameters of dairy cows in peripartum period and early lactation. Mljekarstvo 62(2):151-158.

Vannucchi, C. I., J. A. Rodrigues, L. C. Silva, C. F. Lúcio, G. A. Veiga, P. V. Furtado, C. A. Oliveira, and M. Nichi. 2015. Association between birth conditions and glucose and cortisol profiles of periparturient dairy cows and neonatal calves. Vet Rec 176(14):358.

Wankhade, P. R., A. Manimaran, A. Kumaresan, S. Jeyakumar, K. P. Ramesha, V. Sejian, D. Rajendran, and M. R. Varghese. 2017. Metabolic and immunological changes in transition dairy cows: A review. Vet World 10(11):1367-1377. 